\title{
IJIDI - The New Beginning: A Welcome Editorial
}

\author{
Keren Dali and Paul T. Jaeger
}

Keywords: diversity; inclusion; peer review; publishing; scholarly communication

Publication Type: editorial

\section{Editorial}

$\mathrm{T}$

here will likely be some people who throw their hands up and roll their eyes, thinking to themselves-"Another journal about diversity." And yet, IJIDI is not just "another journal." The overuse of the word diversity is undeniable, and has served to undermine not only its own true meaning and value, but also the value of the related concepts of social justice and inclusion. Amid the multiplicity of venues that discuss diversity, it is also easy to overlook the fact that there is actually no scholarly or professional journal solely or chiefly dedicated to diversity and inclusion in LIS. This is all the more reason, however, to have a venue that takes a fresh unorthodox look at diversity and opens up conversations that are covered by an expanded spectrum of topics, formats, and contributing disciplines. Such a venue promotes the editorial culture that privileges diversity and creativity of expression and safeguards them from constricting conventions and the political correctness of academia, while maintaining scholastic and methodological rigor, excellence in production and design, and high writing standards.

The idea for this journal came from the annual Conference on Inclusion and Diversity in Library \& Information Science (CIDLIS). For the past seven years, CIDLIS (initially called Symposium on Diversity and Inclusion in Library \& Information Science Education) has annually featured dozens of presentations that highlight innovative projects, services, and practices, disseminate new research findings, and discuss major issues that need to be addressed. Beyond the conference, however, both CIDLIS presenters and attendees have noted difficulties in finding good homes for their papers. This made sense in light of the noted shortage of specialized diversity journals and the fact that general interest LIS journals can only include a limited number of diversity papers, as afforded by their missions and scopes. So, the solution seemed to be creating an online journal devoted to this area.

The past year has seen the journal finding its bearings and defining its course, and it is now emerging with a new and clear direction and a new editorial team made up of scholars and practitioners who live their scholarship. In 2018, we are starting a new chapter, beginning with two combined special issues based on the presentations shared at the Diversity by Design Symposium in Toronto, ON, Canada, held in September of 2017.

IJIDI promotes an image and concept of diversity that goes beyond demographic characteristics. While the importance of continuous discussions about the diversity of participation and representation in the context of race, ethnicity, language, gender, social class, and body ability cannot be understated, we are guided by recently emerged ideas, including values-based diversity and diversity by design, and also by a combination of 
approaches to diversity emanating from the academy and the corporate sector. We are committed to micro- and macro-level discussions and value the policy focus as much as we value attention to individuals, groups, and humanistic concerns. We position diversity as a matter that touches every member of the public and not only minority groups. The social justice approach to diversity is complemented by the vision of diversity as an engine of prosperity and social progress, without which harmonious co-existence and economic growth are not possible.

A unique aspiration of our journal is to provide international coverage of diversity issues and a wider forum for voices from outside of North America and the English-speaking world. The word "information" in the title opens a door that connects IJIDI to allied professional fields and cognate disciplines, including contributions from museums, archives, performing arts, and other cultural institutions, computer science, health, disability studies, and social work, among others.

The journal encourages the submission of a variety of publication types: original empirical research; conceptual and theoretical papers; professional experience-sharing articles; viewpoint submission; conference reports; and shorter miscellaneous pieces. We particularly welcome contributions from emergent scholars, graduate students, and young professionals. It is important for IJIDI to grow its own authorship base and to increase the numbers of those who champion scholarly and professional communication on diversity issues.

Let's face it: many of us like to engage in discussions of diversity in various settings. Yet, when it comes to writing and publishing about diversity, we usually hear from a small and limited number of voices, with the same names filling up publication venues. To make a true impact, we need more than just conference talks and panels. If we are serious about making a difference in the state of diversity and inclusion, we need a broader reach; we need the power of the published statement; we need the clarity and persuasiveness of well-formulated ideas. Publishing is not the prerogative of a select few. If you have brave and creative ideas and an open mind, if you dream of making diversity conversations meaningful and global, if you're committed to diversity scholarship, innovation in professional practices, or motivational speaking-the IJIDI team is here to help you get your research, experience, and message across.

We realize full-well that the road to championing the cause is a bumpy one, notwithstanding the fact that education and engagement for people from diverse backgrounds and fostering a more inclusive society is the beating heart of the LIS field. Too often, our efforts in these areas are not widely shared, and innovative approaches and key lessons learned go undocumented. IJIDI ensures that there is a venue for sharing and learning from diversity and inclusion initiatives, programs, teaching, and research around the globe and between professionals, educators, and students. Through IJIDI, we can collaboratively learn, improve, and grow our efforts in facilitating and encouraging the development of a more just and equitable world.

As an open-access online journal, IJIDI will have the capacity to accommodate innovative formats involving supplementary files, full-text creative writing, visual production, and multimedia. Our 2018 special issues will be an example thereof.

The journal fosters the culture of prompt and respectful double-blind peer reviewing. You can read more about our editorial policies and peer review process on the journal website.

Starting 2018, the journal will feature a book review section. This section will be headed by the team of an academic and a public librarian, and will include not only reviews of scholarly monographs on diversity but also reviews of diversity fiction. It is our hope that the community of practitioners from all types of libraries and information organizations will become not only 
readers but also contributors to this section, defining its direction and content.

We invite submissions from the members of the LIS community and cognate disciplines and encourage everyone to think about diversity and inclusion in the broadest possible terms. This, we believe, will help many researchers to see their work through the diversity lens, to find its relevance to the mission and scope of $I J I D I$, and to engage in a dynamic and open conversation through the venue that is not "just another journal about diversity."

\section{Acknowledgements}

We thank wholeheartedly our dedicated international team of Managing Editors whose enthusiasm, creativity, and indefatigable work have made these issues possible. They are the backbone of the journal and the heart of our team. We also thank IJIDI reviewers who have done an amazing job delivering timely, expert, and nuanced reviews amid their busy schedules and often on tight deadlines to accommodate the production schedule of special issues. Their support is invaluable to the journal, and we hope they will continue taking this journey with IJIDI in the future. We thank our copyeditors; colleagues from other journals who consulted us in the process; and the UMD library team who continue to provide technical guidance and support to IJIDI. Last but not least, we thank our authors for their original contributions to advance the diversity conversation.

Dr. Keren Dali's (keren.dali@alumni.utoronto.ca) research interests are in diversity and marginalized communities, relationships between LIS and Social Work; disabilities; LIS education with the focus on creativity, accreditation, and humanistic pedagogies; and reading behaviors. With the background in Social Work and LIS, Dr. Dali holds the inaugural Outstanding Instructor Award from the Faculty of Information, University of Toronto; the inaugural ALISE/Connie Van Fleet Award for Research Excellence in Public Library Services to Adults; the Outstanding Reviewer distinction and the Outstanding and Highly Commended Paper distinctions from the Emerald Literati Network Awards for Excellence. Her work has been funded by the grants from the Social Sciences \& Humanities Research Council of Canada (SSHRC) and the American Library Association, among others. She's active in ASIS\&T, ALISE, and IFLA, chairing committees for both ASIS\&T and ALISE.

Dr. Paul T. Jaeger (pjaeger@umd.edu) is Professor, Diversity Officer, and Director of the MLIS program of the College of Information Studies and Co-Director of the Information Policy and Access Center (iPAC) at the University of Maryland. His teaching and research focus on the ways in which law and public policy shape information behavior, with a specific focus on issues of human rights and social justice. His research has been funded by the Institute of Museum \& Library Services, the National Science Foundation, the American Library Association, the Smithsonian Institution, and the Bill \& Melinda Gates Foundation, among others. Dr. Jaeger is a founder and chair of the Conference on Inclusion and Diversity in LIS and of the UMD Disability Summit. He is the author of more than 160 journal articles and book chapters, as well as more than a dozen books, and is a recipient of the Library Journal/ALISE Excellence in Teaching Award. 